Interactive comment on "The Rofental: a high Alpine research basin ( $1890 \mathrm{~m}-3770 \mathrm{~m}$ a.s.l.) in the Ötztal Alps (Austria) with over 150 years of hydro-meteorological and glaciological observations” by Ulrich Strasser et al.

Ulrich Strasser et al.

ulrich.strasser@uibk.ac.at

Received and published: 5 November 2017

\title{
Author comment on
}

Interactive comment on "The Rofental: a high Alpine research basin (1890 m - 3770 $\mathrm{m}$ a.s.l.) in the Ötztal Alps (Austria) with over 150 years of hydro-meteorological and glaciological observations" by Ulrich Strasser et al.

A. Winstral (Referee) 
Review of the manuscript "The Rofental: a high Alpine research basin (1890 m - 3770 $\mathrm{m}$ a.s.I.) in the Ötztal Alps (Austria) with over 150 years of hydrometeorological and glaciological observations" by Strasser et al., 2017.

Interactive

comment

Thank You very much for Your valuable review. All Your suggestions contribute to the improvement of our work. In the preparation of a revised version of the manuscript we consider Your specific comments as follows:

This manuscript describes much of the data available from the glaciated Rofental research basin in Austria. Data collection in this basin is still quite active. Current activity along with a historic glacier database dating back over 150 years provides for a valuable resource of publicly available data. Much of these data are already available and I commend the authors for this. I look forward to a worthwhile companion paper introducing researchers to this valuable collection. Unfortunately, I find the current manuscript would greatly benefit from some major revisions. As this is just a data paper all that the revisions really entail is a thorough rewrite and reorganization that hopefully is not too time consuming.

We have already done significant re-writing and re-organizing according to the comments of reviewers 1-3. We acknowledge Your additional comments as further step to improve the manuscript in its revised form

I find the current work to be a very difficult read. There are numerous grammatical errors and poorly constructed sentences and paragraphs. Aside from these points, I also found the work to be poorly organized. It seems as though a bunch of facts are just thrown at the reader in a non-systematic fashion. I realize it can be hard to "tell a story" in a data paper, but I think there is one here that should be exploited. In my view there is a remarkable set of historic glacier data here that is then embellished in the 
more recent years with meteorological data, runoff data, and now LiDAR. I think that is the story: a) historic glacier data showing changes; b) now a bunch of meteorological data and runoff data to better connect the climate and hydrology to the glacier activity have been collected; and c) higher temporal and spatial resolution glacier data are now available from laser scanning. Organizing the work in this manner also provides a general timeline to the presentation rather than as currently set up where the data are presented as met -> glacier -> LiDAR. Once you have a general story, I think each subsection should start out describing each particular set of data and then how they have contributed (with references) or could contribute to our scientific understanding while also making connections back to the general story.

We have re-organized the order of the data to follow Your suggestion of a more attractive story; the long glacier monitoring history of the site is now in the beginning, including a new illustration. We also have added a figure showing the periods covered by all available data. Finally, the usability of the data is briefly described in the respective subsections. And we have stressed the fact that - with respect to the purpose of an INARCH spec. iss. - we have concentrated our efforts to provide (i) a mostly complete picture of the water balance components of the Rofental - the mass balances of the observed glaciers being an important highlight of these - and (ii) the meteorological data to force a typical hydrological catchment model

Another key point that I found somewhat odd is that a large amount of the text is spent describing data that will be collected in the future or was just recently collected as this paper was being written. I think a data paper should focus on what's been collected and what is currently available to the readers. It is fine to mention future work, but too much of the paper seems to be appropriated to these types of "future" data. There is plenty of historical data available for this site. I think it would be better to spend more time on the data that has been collected and that is currently available to the public.

We remove respective outlooks to what will be provided in the future to the necessary. Instead, we include a brief info ot what the "ESSD livinig data process" means 
Some points illustrating my concerns: âǍíThe introduction should get the reader excited and prepared for the subsequent presentation. This is a wonderful dataset and ESSDD there is enough quality data here to do just that. I think this section could benefit from a rewrite.

We re-write the introduction and add substantial material to the long history of the glacier monitoring in the Rofental, including new illustrations

Interactive

comment

The introduction begins with a paragraph that consists of a single run-on sentence mentioning the many institutions involved in the Rofental. The opening paragraph should highlight why these data are so important and get the readers excited. A listing of institutions just doesn't do this. The current opening paragraph is secondary to the main point of the paper, which is data, and should come towards the end of the introduction.

We add an explanation of the scientific meaning and potential use of the data provided for the Rofental. And we have already shifted the current opening paragraph towards the end of the introduction, according to Your suggestion

Second paragraph of introduction. The third sentence in this paragraph is the main point of this paragraph and maybe the main point of this entire paper: "The glacier mass balance time series of Hintereis-, Vernagt- and Kesselwandferner (HEF, VF and KWF further on) are among the longest uninterrupted series worldwide". As such, this sentence should be the first sentence in the paragraph followed by the other sentences that further describe and detail these data. It is not clear how the final sentence of this paragraph connects to any of the others here.

We have re-strucured also the second paragraph of the introduction

Fourth and final paragraph of introduction. This is one example of what I feel is "poor paragraph structure" and a small-scale example of the prevalent feeling of being presented with random facts without any sensible story. The first two sentences describe 
the collection of airborne LiDAR data and what these data have been used for. The third sentence extends the ALS description to TLS data, which is fine. So as a reader at ESSDD this point I'm expecting this paragraph to be about LiDAR data. But then from the latter part of this sentence where the conversation drifts to turbulent flux measurements, the reader just gets hit by a series of seemingly unorganized factoids with no connection to what appeared to be the main point of the paragraph. We get a sentence reporting on where key glaciological results can be found, which is followed by a sentence stating that two research stations in the basin serve as logistic bases for fieldwork. Then a sentence on the fact that several mountain huts are located in the Rofental. The paragraph's concluding sentence tells the reader that the highest permanently settled mountain farm in Austria is also in the study area. I'm not sure what the main point of this paragraph was intended to be.

We are re-strucuring also the fourth and final paragraph of the introduction to give them consistent main points that are described

I think amongst other things it would be beneficial for readers if the authors concluded their introduction with an outline of what is going to be subsequently presented and in what order.

We add such an outline, including an illustration of what is available

Figure 2 would benefit from larger, better differentiated symbols, an outline of the Vernagtbach watershed, and an inset map that could place the Rofental in relationship to Austria/Europe.

We have updated the map accordingly

Data from the Hintereis- and Kesselwandferner are continually presented together, but the reader is never guided as to why they are treated this way.

We will add an explanation

Section 3.1.1 Hintereis- and Kesselwandferner. I can't see where any data from the 
Kesselwandferner are described here. Two paragraphs appear in this sub-section: one describing Hintereis data and another describing "future" (installed 2017) data. I'm also not sure that latitude/longitude data should be included in the text as they do not provide the reader with any usable information at this point. I think location data should just be in the header of the data files or maybe in a ReadMe text file that accompanies the data. I also think it's best to set up these sub-sections as first describing these data and then descriptions of how these data have been used (with references) or could be used.

We will include KWF here. As for the latitude/longitude data we suggest to leave it in the text for data users who are happy to find this all available in the paper text of course, the metadata is also available in PANGAEA where the files are provided for download. Finally, we will re-arrange the subsections in the text according to Your suggestion

When I went to PANGEA website, meteorological data for Hintereis-, Kessel-, and Vernagtferner were only available through 2012 . Based on what is presented in this manuscript I expected the data would be more current. I think before going to press that data should either be updated on the website/repository or the fact that publiclyavailable data is only current through 2012 should be mentioned in the manuscript.

We mention it now, we refer to the living data process and we have included a comprehensive figure showing what is available for which period

Section 3.1.3. The last two paragraphs here describe the data from two high-elevation sites. These data are important due to this fact yet this point isn't made until about the fourth sentence of the last paragraph. Mention the importance at the beginning then describe these data. I also recommend at this point touching back to how these data help capture the steep environmental gradients mentioned in the introductory paragraph of this section (3.1). This is an example of how I think one should connect back to the "story".

Interactive comment
Printer-friendly version

Discussion paper 
We do mention it now in the beginning, and then describe the data. We also refer back to the introductory paragraph of this section, i.e. connect back to the "story"

Section 3.4. It is stated that laser scanning data provides spectral information. I don't believe this is a correct statement.

Yes, there is spectral information. We have further specified "there is additional information on the spectral properties intensity and reflectance of the scanned surface in the wavelength of the specific scanner"

Figure 7. I had a lot of difficulty deciphering this figure. What exactly is "accumulation" as used here? What are the acronyms that appear in the figure? I believe "Glaciological balance" refers to the calculations based on the laser data but that hasn't been made clear.

We will make an attempt to improve the figure and its caption; it is, however, not yet finally decided if we leave the figure in the manuscript or not

Section 5. Conclusions and outlook. If there is a conclusive sentence here, I couldn't find it. There are three sentences in this section. The first one is a run-on sentence (5+ lines and well over 60 words) describing several projects the Rofental is part of. This sentence should probably appear in the introduction. The second sentence states the Hintereis station is part of another larger grouping. This also seemingly belongs in the Introduction or in the site descriptions. The third sentence does fit under the heading "outlook". My scorecard shows zero conclusive sentences and one on outlook. This needs a rewrite.

We are re-writing the conclusions section according to the data provision character of the paper, and use the information in the sentences not needed here in the appropriate sub-sections

Thank You! 
Interactive comment on Earth Syst. Sci. Data Discuss., https://doi.org/10.5194/essd-2017-85, 2017.

\section{ESSDD}

Interactive comment 